\title{
Ambulatory consolidation chemotherapy for acute myeloid leukemia with antibacterial prophylaxis is associated with frequent bacteremia and the emergence of fluoroquinolone resistant $\mathrm{E}$. Coli
}

\author{
Lalit Saini ${ }^{1}$, Coleman Rostein ${ }^{2}$, Eshetu G Atenafu ${ }^{3}$ and Joseph M Brandwein ${ }^{1 *}$
}

\begin{abstract}
Background: Ambulatory consolidation chemotherapy for acute myeloid leukemia (AML) is frequently associated with bloodstream infections but the spectrum of bacterial pathogens in this setting has not been well-described.

Methods: We evaluated the emergence of bacteremias and their respective antibiotic susceptibility patterns in AML patients receiving ambulatory-based consolidation therapy. Following achievement of complete remission, 207 patients received the first cycle (C1), and 195 the second cycle (C2), of consolidation on an ambulatory basis. Antimicrobial prophylaxis consisted of ciprofloxacin, amoxicillin and fluconazole.

Results: There were significantly more positive blood cultures for E. coli in C2 as compared to C1 (10 vs. 1, $\mathrm{p}=0.0045$ ); all E. coli strains for which susceptibility testing was performed demonstrated resistance to ciprofloxacin. In patients under age 60 there was a significantly higher rate of Streptococccus spp. bacteremia in C2 vs. C1; despite amoxicillin prophylaxis all Streptococcus isolates in C2 were sensitive to penicillin. Patients with Staphylococcus bacteremia in $\mathrm{C} 1$ had significantly higher rates of Staphylococcus bacteremia in C2 ( $p=0.009, \mathrm{OR}=8.6)$.

Conclusions: For AML patients undergoing outpatient-based intensive consolidation chemotherapy with antibiotic prophylaxis, the second cycle is associated with higher rates of ciprofloxacin resistant $E$. coli, penicillin-sensitive Streptococcus bacteremias and recurrent Staphylococcus infections.
\end{abstract}

Keywords: Acute myeloid leukemia, Chemotherapy, Infections, Bacteremia, Antibiotic resistance

\section{Background}

Intensive treatment for acute myeloid leukemia (AML) typically includes induction followed by $2-4$ cycles of consolidation chemotherapy [1]. In recent years there has been a shift to outpatient-based AML consolidation therapy with a number of studies confirming the safety of this approach for selected patients [2-9].

In most centers ambulatory chemotherapy programs rely on the use of antimicrobial prophylaxis as a means

\footnotetext{
* Correspondence: joseph.brandwein@uhn.ca

'Department of Medical Oncology and Hematology, Princess Margaret Hospital, University of Toronto, 610 University Avenue, Rm. 5-109, Toronto, ON M5G 2M9, Canada

Full list of author information is available at the end of the article
}

of reducing infections. Recent Infectious Disease Society of America (IDSA) guidelines endorse the use of ciprofloxacin prophylaxis to reduce bacterial infections in high risk cancer patients receiving chemotherapy [10]. Despite prophylactic antibiotics $30-90 \%$ of ambulatory patients experience a fever and $20-90 \%$ of consolidation cycles are associated with hospitalizations [2,4-6]. However, there are limited data on the characteristics of infectious complications in this setting. We have previously shown that infectious complications increase in the second cycle of ambulatory AML consolidation therapy resulting in greater need for intensive care unit (ICU) support and increased mortality [11]. As these findings may relate to breakthrough infections caused by

\section{Ciomed Central}


the development of antibiotic resistant microbes despite prophylactic antibiotic use, we conducted a retrospective analysis evaluating the spectrum of microbiological isolates and their antibiotic resistance patterns in AML patients undergoing ambulatory-based consolidation chemotherapy.

\section{Methods \\ Patients}

All newly diagnosed AML patients at the Princess Margaret Hospital (PMH) who had achieved complete remission with frontline induction chemotherapy and proceeding to ambulatory-based consolidation chemotherapy from October 2002 - February 2008 were included in the analysis. Eligibility requirements were as previously described [11]. Patients received the second consolidation cycle following peripheral count recovery from the first cycle and resolution of any previous bacterial infections. Prior approval for the study was obtained from the Cancer Registry Data Access Committee and Research Ethics Board of the University Health Network, Toronto, Ontario.

\section{Chemotherapy regimens and supportive care}

Ambulatory chemotherapy, subsequent monitoring and supportive care were provided as previously described [11] either in the ambulatory care area of the PMH or a local community hospital/clinic. Patients under age 60 (younger cohort) received two cycles of consolidation therapy ( $\mathrm{C} 1$ and $\mathrm{C} 2$ ), each consisting of high dose cytarabine (HiDAC) 3 grams $/ \mathrm{m}^{2}$ IV q12 hours $\times 6$ doses on days 1,3 , and 5 , plus daunorubicn $45 \mathrm{mg} / \mathrm{m}^{2}$ IV daily on days 1 and 2 . For patients over age 60 (older cohort), C1 consisted of daunorubicin $60 \mathrm{mg} / \mathrm{m}^{2}$ IV daily $\times 3$ plus cytarabine $100 \mathrm{mg} / \mathrm{m}^{2}$ as a 7-day continuous infusion via ambulatory infusion pump and $\mathrm{C} 2$ consisted of mitoxantrone $10 \mathrm{mg} / \mathrm{m}^{2}$ IV plus etoposide $100 \mathrm{mg} / \mathrm{m}^{2}$ IV, each given once daily from days $1-5$.

During induction therapy patients received antimicrobial prophylaxis with fluconazole $400 \mathrm{mg}$ PO daily whereas during consolidation they received ciprofloxacin $500 \mathrm{mg}$ PO q12 hours, amoxicillin $500 \mathrm{mg}$ PO q8 hours and fluconazole $400 \mathrm{mg}$ PO daily, starting on Day 8 of the chemotherapy cycle and continuing until absolute neutrophil count $\left(\right.$ ANC) $>0.5 \times 10^{9} / \mathrm{L}$. All chemotherapy, transfusions and IV antibiotics were administered via central venous catheters $(\mathrm{CVC})$, usually double lumen Hickman lines placed prior to the start of induction chemotherapy.

Patients presenting with fever or clinical features of an infection had blood cultures drawn from all CVC ports, a peripheral vein and any other accessible sites suspected of being infected. As per institutional policy, empiric antibiotics for febrile neutropenia most commonly consisted of pipracillin/tazobactam 4.5 grams IV q8 hours plus tobramycin $5 \mathrm{mg} / \mathrm{kg}$ IV q24 hours. Febrile patients with suspected CVC involvement i.e. those with erythema, pain or purulent drainage at the insertion site, were started on vancomycin 1 gram IV q12 hours. Empiric therapy was adjusted based upon the results of culture and sensitivity testing. Antimicrobials were continued until microbiological and clinical evidence of infection had resolved and the ANC was $>0.5 \times 10^{9} / \mathrm{L}$. Hematopoietic growth factors were not routinely used but were administered at the physician's discretion from day 16-18 until neutrophil recovery. Patients with blood cultures positive for coagulase negative staphylococci (CNST) usually retained their CVC whereas patients with candidemia, tunnel infections or those with persistently positive cultures beyond 72 hours had their CVC replaced.

Cultures were processed using standard microbiological methods. Antibiotic susceptibilities were performed and reported by the Department of Microbiology of the Mount Sinai Hospital/University Health Network (Toronto, Ontario) or the local community hospital (if the site of the patient's initial presentation) using predefined criteria. Antibiotic sensitivity data were not available in some cases where patients presented to local community hospitals.

\section{Definitions}

Blood cultures were considered positive if one or more culture bottles showed growth, regardless of the organism, with symptoms compatible with an infection. Bacteremias related to common contaminants such as CNST, Micrococcus., Propionibacterium or Bacillus spp. were treated with a full course of antibiotics; thus no distinction was made between contaminants and true pathogens. Cultures were considered polymicrobial if at least two different organisms were isolated. Repeated cultures in persistently febrile patients yielding the same organism with the same antibiotic susceptibility profile were considered as one isolate unless separated by one or more negative blood cultures.

\section{Statistical analysis}

Descriptive statistics were used to describe patient characteristics and outcome recordings. Categorical variables were expressed as count and proportions whereas continuous variables were expressed as means. Pearson Chisquare or Fisher's exact test, when applicable, were used when comparing rates of infection and readmission. Results were considered significant if two-tailed $\mathrm{P}$ was $<0.05$. Statistical Analysis Software (SAS, version 9.2; SAS Institute Inc., Cary NC) was used for statistical analysis.

\section{Results}

\section{Patient characteristics}

The characteristics of the patients receiving ambulatorybased consolidation chemotherapy have been previously 
described [11], and are summarized in Table 1. There was no significant difference in the median age of patients between $\mathrm{C} 1$ and $\mathrm{C} 2 ; 54.6 \%$ of patients in $\mathrm{C} 1$ and $52.3 \%$ in $\mathrm{C} 2$ were male and the proportion of patients age $\geq 60$ was $30 \%$ in $\mathrm{C} 1$ and $33 \%$ in $\mathrm{C} 2(\mathrm{p}=\mathrm{NS})$.

\section{Bacteremia during ambulatory chemotherapy}

Microbiologically documented episodes of bacteremia are shown in Table 1. As we previously reported, the ambulatory cohort in $\mathrm{C} 2$ had significantly higher rates of positive blood cultures and bloodstream infection relative to $\mathrm{C} 1$ [11]. Most patients had only a single positive blood culture (86\% in $\mathrm{C} 1$ and $90 \%$ in C2) with greater than $90 \%$ of cultures in each cycle being monomicrobial. In each consolidation cycle significantly more patients had a gram-positive bacterial infection as compared to gram-negative infections. The proportion of gram positive vs. gram negative isolates was not significantly different in $\mathrm{C} 1$ as compared to C2. Approximately $30 \%$ of patients presented to local community hospitals, where the source of the positive blood culture (CVC vs. peripheral) was not identified; we were therefore unable to determine the extent of CVC related bacteremias in these cases.

As shown in Table 1, CNST were the most common gram positive isolates during both cycles. Streptococci constituted $17 \%(9 / 53)$ of the total isolates in $\mathrm{C} 1$ and $23.9 \%(17 / 71)$ of the total isolates in $\mathrm{C} 2(\mathrm{p}=0.35)$. Younger patients had a significantly higher incidence of streptococcal bacteremia in $\mathrm{C} 2$ relative to $\mathrm{C} 1(11.2 \%$ vs. $4.3 \%, \mathrm{p}=0.033$ ), and in these patients streptococci represented a greater proportion of the total isolates in C2 (34\%, $15 / 44$ isolates) relative to $\mathrm{C} 1(15 \%, 6 / 40$ isolates, $\mathrm{p}=0.044)$.

Gram negative organisms were identified in $5.6 \%$ of patients in $\mathrm{C} 1$ vs. $10.7 \%$ of those in $\mathrm{C} 2(\mathrm{p}=0.07)$. The frequency of $E$. coli isolates was significantly higher in $\mathrm{C} 2$ relative to $\mathrm{C} 1(5.4 \%$ vs. $0.5 \%, \mathrm{p}=0.005)$ with $E$. coli constituting 14.1\% (10/71) of all isolates in C2 and 1.9\% (1/53) of those in $\mathrm{C} 1 \quad(\mathrm{p}=0.023)$. This trend was observed in both the younger (0 in $\mathrm{C} 1$ vs. 4 in $\mathrm{C} 2$ ) and older (1 in $\mathrm{C} 1$ vs. 6 in C2) cohorts. In addition, there was no difference in the incidence of $E$. coli bacteremia in younger versus older patients within $\mathrm{C} 1(\mathrm{p}=0.827)$ or $\mathrm{C} 2(\mathrm{p}=0.8)$. The proportion of patients with non-E. coli gram negative isolates did not differ between $\mathrm{C} 1$ and $\mathrm{C} 2$ (5.1\% vs. $5.3 \%, \mathrm{p}=0.91$ ).

\section{Antibiotic resistance patterns}

Table 2 highlights the antibiotic resistance patterns for selected organisms. Only one of the CNST with antibiotic sensitivity data was resistant to vancomycin while virtually all isolates were resistant to cloxacillin. All Streptococcus spp. isolates with susceptibility data exhibited sensitivity to penicillin. Among the non- E. coli gram-negative isolates, there was no difference in rates of ciprofloxacin resistance between $\mathrm{C} 1$ and $\mathrm{C} 2$ (1/9 vs. 0/7, p=1.0). In contrast, all E. coli isolates $(8 / 8)$ from patients in $\mathrm{C} 2$ for which susceptibilities were available were ciprofloxacin resistant.

As shown in Table 2, most gram negative isolates in $\mathrm{C} 1$ and $\mathrm{C} 2$ were sensitive to the commonly used empiric antibiotics for febrile neutropenia in our institution, piperacillin/tazobactam and aminoglycosides. However, tobramycin resistance was noted in $67 \%(4 / 6)$ of the E. coli isolates, including $3 / 5$ isolates from patients in $\mathrm{C} 2$.

\section{Infection-related mortality}

Overall, 9 patients died due to infectious complications while receiving ambulatory chemotherapy, one patient during $\mathrm{C} 1$ and 8 during $\mathrm{C} 2$. CNST were isolated from one such patient during C2 whereas Streptococcus spp. was isolated from two patients who died (one each in C1 and C2). The single Streptococcus isolate with available antibiotic susceptibility information was sensitive to penicillin and vancomycin. Gram negative organisms were isolated in 6 of the 8 patient deaths in C2: Klebsiella spp. (3 cases), E. coli (2) and Pseudomonas spp. (1, urine isolate). Both $E$. coli isolates were resistant to ciprofloxacin and tobramycin and displayed sensitivity only to piperacillin/tazobactam. Mortality amongst patients with ciprofloxacin resistant gram-negative bacteremias was $20 \%(2 / 10)$ versus $13.3 \%(2 / 15)$ for those with ciprofloxacin sensitive strains $(\mathrm{p}=1.0)$.

\section{Effect of previous positive cultures/hospitalization}

Approximately $51 \%$ of patients with a positive blood culture in $\mathrm{C} 1$ had a subsequent positive culture in $\mathrm{C} 2$ while only $27 \%$ of those with negative blood cultures in $\mathrm{C} 1$ had a positive culture in $\mathrm{C} 2(\mathrm{OR}=2.8, \mathrm{p}=0.005)$. This trend was significant for gram-positive organisms $(\mathrm{OR}=3.25, \mathrm{p}=0.004)$ but not for gram-negative organisms $(\mathrm{OR}=2.1, \mathrm{p}=0.31)$. An infection with Staphylococcal isolates in $\mathrm{C} 1$ was associated with the highest recurrence rates in $\mathrm{C} 2$ but only for patients age $<60$ $(\mathrm{OR}=8.6, \mathrm{p}=0.009)$. No such association was seen for Streptococcal isolates or in the older cohort.

\section{Discussion}

Recent data suggest that ambulatory chemotherapy for AML patients can reduce the incidence of septicemia [7]. However, published data on the characteristics of microbiological isolates from patients receiving ambulatory chemotherapy remains limited [2-7,9]. Our study, focusing on bacterial bloodstream infections, represents the largest reported series on AML patients receiving ambulatory-based intensive consolidation chemotherapy. We have previously shown that the rate of positive blood cultures increases following the second consolidation 
Table 1 Blood culture results

\begin{tabular}{|c|c|c|c|}
\hline & C\#1 & $\mathrm{CH2}$ & (p-value) \\
\hline Total Number of Patients & 196 & 187 & \\
\hline Age $<60$ & 140 & 126 & \\
\hline Age $\geq 60$ & 56 & 61 & \\
\hline$\%$ Male & $107(54.6 \%)$ & $98(52.3 \%)$ & \\
\hline At least one blood culture positive (\% of patients) & $43(21.9 \%)$ & $59(31.6 \%)$ & $P=0.03$ \\
\hline 1 culture positive & $37(86 \%)$ & $53(90 \%)$ & NS \\
\hline 2 or more cultures positive & $6(14 \%)$ & $6(10 \%)$ & NS \\
\hline Patients with Gram Positive isolates (\% of Patients) & $35(17.9 \%)$ & $46(24.6 \%)$ & 0.11 \\
\hline Patients with Gram Negative isolates (\% of patients) & $11(5.6 \%)$ & $20(10.7 \%)$ & 0.07 \\
\hline Gram Positive vs. Gram Negative & $P=0.0002$ & $P=0.0004$ & \\
\hline Total number of positive blood cultures: & 49 & 65 & \\
\hline Monomicrobial cultures (\% of total cultures) & $45(91.8 \%)$ & $60(92.3 \%)$ & NS \\
\hline Polymicrobial cultures (\% of total cultures) & $4(8.2 \%)$ & $5(7.7 \%)$ & NS \\
\hline Total number of isolates: & 53 & 71 & \\
\hline Age $<60$ & 40 & 44 & \\
\hline Age $\geq 60$ & 13 & 27 & \\
\hline \multicolumn{4}{|l|}{ Clinical Isolates (\% of patients) } \\
\hline Gram Positive $^{+}$ & $35(17.9 \%)$ & $46(24.6 \%)$ & $P=0.10$ \\
\hline$C N S T^{*}$ & $19(9.7 \%)$ & $23(12.4 \%)$ & NS \\
\hline Corynebacterium & $5(2.6 \%)$ & $2(1.1 \%)$ & \\
\hline Micrococcus & $4(2.0 \%)$ & & \\
\hline Enterococcus & $1(0.5 \%)$ & $3(1.6 \%)$ & \\
\hline Staphyloccoccus. aureus & $2(1.0 \%)$ & $1(0.5 \%)$ & \\
\hline Streptococcus species & $9(4.6 \%)$ & $16(8.6 \%)$ & $P=0.11$ \\
\hline Streptococcus viridins & $6(3.1 \%)$ & $13(7.0 \%)$ & \\
\hline Streptococcus viridians/Streptococcus oralis & - & $1(0.5 \%)$ & \\
\hline Streptococcus mitis & $2(1.0 \%)$ & - & \\
\hline Streptococcus auralis & - & $1(0.5 \%)$ & \\
\hline Streptococcus spp. & $1(0.5 \%)$ & $1(0.5 \%)$ & \\
\hline < age 60 with Strep. & $6(4.3 \%)$ & $14(11.2 \%)$ & $P=0.033$ \\
\hline$\geq$ age 60 with Strep. & $3(5.36 \%)$ & $2(3.3 \%)$ & NS \\
\hline Other Gram Positive & $1(0.5 \%)$ & $4(2.1 \%)$ & \\
\hline Gram Negative & $11(5.6 \%)$ & $20(10.7 \%)$ & $P=0.07$ \\
\hline Age $<60$ & $8(5.7 \%)$ & $9(7.1 \%)$ & NS \\
\hline Age $\geq 60$ & $3(5.4 \%)$ & $11(18 \%)$ & $P=0.035$ \\
\hline Eschericia coli & $1(0.5 \%)$ & $10(5.3 \%)$ & $P=0.005$ \\
\hline Klebsiella & $2(1.0 \%)$ & $5(2.7 \%)$ & \\
\hline Enterobacter & $3(1.5 \%)$ & $1(0.5 \%)$ & \\
\hline Pseudomonas spp. & $4(2.0 \%)$ & - & \\
\hline Other Gram Negative & $1(0.5 \%)$ & $4(2.1 \%)$ & \\
\hline Yeast & - & $1(0.5 \%)$ & \\
\hline
\end{tabular}

$\mathrm{CNST}^{*}$ = coagulase-negative staphylococci;

Gram Positive $^{+}=$Species information available for 185 pts. in C2 (Age $<60=125$, Age $\geq 60=65$ ). 
Table 2 Antibiotic resistance patterns of isolates (number showing resistance / isolates with antibiotic sensitivity data available)

\begin{tabular}{|c|c|c|}
\hline & $\begin{array}{c}\text { Consolidation } 1 \\
\text { (\% of isolates) }\end{array}$ & $\begin{array}{c}\text { Consolidation } 2 \\
\text { (\% of isolates) }\end{array}$ \\
\hline CNST & $20(37.7 \%)$ & $23(32.4 \%)$ \\
\hline Vancomycin resistant & $1 / 15$ & $0 / 21$ \\
\hline Cloxacillin resistant & $14 / 17$ & $21 / 22$ \\
\hline Streptococcus species & $9(17 \%)$ & $17(23.9 \%)$ \\
\hline Penicillin resistant & $1 / 7$ & $0 / 11$ \\
\hline Vancomycin resistant & $0 / 3$ & $0 / 6$ \\
\hline Gram Negatives & $11(20.7 \%)$ & $20(28.1 \%)$ \\
\hline Ciprofloxacin resistant & $2 / 10$ & $8 / 15$ \\
\hline $\mathrm{P} / \mathrm{T}^{*}$ resistant & $1 / 10$ & $0 / 14$ \\
\hline Ampicillin resistant & $9 / 9$ & $13 / 15$ \\
\hline Tobramycin resistant & $1 / 8$ & $3 / 10$ \\
\hline Klebsiella species & $2(3.8 \%)$ & $5(7.0 \%)$ \\
\hline Ciprofloxacin resistant & $0 / 2$ & $0 / 4$ \\
\hline $\mathrm{P} / \mathrm{T}^{*}$ resistant & $0 / 2$ & $0 / 4$ \\
\hline Ampicillin resistant & $2 / 2$ & $4 / 4$ \\
\hline Tobramycin resistant & $0 / 2$ & $0 / 3$ \\
\hline Escherichia coli & $1(1.9 \%)$ & $10(14.1 \%)$ \\
\hline Ciprofloxacin resistant & $1 / 1$ & $8 / 8$ \\
\hline $\mathrm{P} / \mathrm{T}^{*}$ resistant & $0 / 1$ & $0 / 8$ \\
\hline Ampicillin resistant & $1 / 1$ & $7 / 8$ \\
\hline Tobramycin resistant & $1 / 1$ & $3 / 5$ \\
\hline Pseudomonas species & $4(7.6 \%)$ & - \\
\hline Ciprofloxacin resistant & $1 / 4$ & - \\
\hline $\mathrm{P} / \mathrm{T}^{*}$ resistant & $1 / 1$ & - \\
\hline Ampicillin resistant & $3 / 3$ & - \\
\hline Tobramycin resistant & $0 / 4$ & - \\
\hline
\end{tabular}

*P/T = Piperacillin/tazobactam.

cycle [11] and the current study highlights potential explanations for the observed increase.

Despite the isolation of ciprofloxacin resistant E. coli in febrile neutropenic patients receiving fluoroquinolone prophylaxis $[7,12,13]$ their use in high risk patients receiving chemotherapy has been supported by large randomized clinical trials and meta-analysis [14-17]. Within our cohort we observed an increased incidence of gramnegative bacteremia and ciprofloxacin resistance as patients transitioned from $\mathrm{C} 1$ to $\mathrm{C} 2$. Ciprofloxacin resistance was observed in $100 \%$ of the E. coli strains in C2, was rarely seen in Pseudomonas spp (1/4 in C1) and was not observed in the remaining Enterobacteriaceae (0/5 in $\mathrm{C} 1$ and $0 / 7$ in $\mathrm{C} 2)$. The preferential development of resistance among $E$. coli strains has been previously reported [18-20]. Cometta et al. noted that, among a cohort of patients receiving prophylactic fluoroquinolones, $28 \%$ of the $E$. Coli isolates were fluoroquinolone resistant while $>90 \%$ of the $P$. aeruginosa and $K$. pneumoniae isolates remained fluoroquinolone sensitive [18]. Kern et al. postulated the decreased virulence of fluoroquinolone resistant $P$. aeruginosa as one explanation for this phenomenon [20]. Prolonged exposure to subtherapeutic concentrations of antibiotics has been associated with the development of antibiotic resistance [21,22]. It is possible that decreased absorption of fluoroquinolones in the setting of chemotherapy-induced mucositis, may have prevented reaching the mutant prevention concentration $[23,24]$ for $E$. coli in our cohort. We were unable to determine whether there was an emergence of resistance to other antibiotics, although the frequency of $E$. coli aminoglycoside resistance in C2 (4/6 cases) is concerning.

The effects of discontinuing prophylactic fluoroquinolones have been investigated in a number of studies. Reuter et al. prematurely stopped a trial of levofloxacin discontinuation due to a significant increase in infectionrelated mortality [25] and Kern et al. noted increased rates of bacteremia and a trend toward increased mortality during a period in which fluoroquinolone prophylaxis was discontinued [12]. A similar increase in bloodstream infections with fluoroquinolone susceptible isolates was seen when fluoroquinolone prophylaxis was discontinued by two Japanese groups although no effects on mortality were noted $[26,27]$. Thus, although the emergence of ciprofloxacin resistance organisms calls into question the value of ciprofloxacin prophylaxis, current evidence and IDSA guidelines support its use in high-risk patients with prolonged ( $\geq 7$ days) and profound neutropenia $\left(\leq 100\right.$ cells $\left./ \mathrm{mm}^{3}\right)[10]$.

The increased use of fluoroquinolone prophylaxis and indwelling CVC's has led to gram-positive organisms as the main cause of bacteremia in immunocompromised patients $[4,6,7,28]$. In our cohort gram-positive organisms constituted $79 \%$ of the isolates during $\mathrm{C} 1$ and $70 \%$ in C2. As some of our patients were followed in community hospitals, we were unable to accurately determine which isolates were CVC related although we speculate that most CNST were of CVC origin. Consistent with previous reports $[2,6,7,28,29]$, CNST and Streptococcus spp. were the most common gram positive organisms. The rate of CNST isolates was not statistically different between $\mathrm{C} 1$ and $\mathrm{C} 2$, however, Streptococcus isolates, particularly $S$. viridans, increased from $\mathrm{C} 1$ to $\mathrm{C} 2$ within the younger cohort, likely reflecting the cumulative effects of HiDAC chemotherapy [11,28,30-32].

As a high frequency of $S$. viridans infections is a welldocumented effect associated with ciprofloxacin prophylaxis [12], patients within our cohort were administered amoxicillin during the neutropenic period. Surprisingly, 
however, all Streptococcus isolates with susceptibility data were found to be penicillin sensitive. Poor compliance with amoxicillin dosing or prior CVC colonization may provide a potential explanation for this observation. Although current IDSA guidelines recommend against the addition of gram-positive coverage to fluoroquinolone prophylaxis, studies have suggested that it may be beneficial in patients at high risk of streptococcal infections including those with severe neutropenia, oral mucositis, bone marrow transplantation and those receiving HiDACbased chemotherapy [33]. From our data, it is unclear if the addition of amoxicillin alone resulted in any substantial benefit; delineation of this would require a prospective randomized study.

An important finding in our study, not previously described, is the increased rate of positive blood cultures in $\mathrm{C} 2$ in patients with previous positive cultures in $\mathrm{C} 1$. This effect was restricted to Staphylococcus spp. in younger patients and may reflect the prolonged use of indwelling catheters. The inadequate activity of ciprofloxacin and amoxicillin against these organisms likely further contributed to their increased pathogenicity [14,29]. It is possible that CVC replacement between consolidation cycles in patients previously positive for Staphylococcus spp. could reduce their recurrence rates, but this requires further study.

Our data have several limitations. This was a retrospective analysis conducted at a single tertiary care cancer center and it is unclear whether results are generalizable to other centers or with different regimens. Second, our rates of gram-positive bacteremia may have been overestimated as we could not always accurately differentiate whether isolates represented true bacteremia, CVC colonization or contamination. However, given the context of fever in high-risk neutropenic patients, all of whom were treated empirically with IV antibiotics, infection is more likely. Furthermore, as some patients were treated in community hospitals, we had incomplete information regarding antibiotic sensitivities.

\section{Conclusions}

In summary, our findings emphasize the limitations of antibacterial prophylaxis of AML patients post-chemotherapy in the ambulatory setting, particularly in later cycles following prolonged use of fluoroquinolones and indwelling central venous catheters. Clinicians should be cognizant of the resulting increased predilection to fluoroquinoloneresistant E. coli, streptococcal spp. and recurrent CNST infections, and additional prophylactic measures should be considered in these patients.

\section{Competing interests}

All authors declare that they have no competing interests.

\section{Authors' contributions}

LS performed the research and drafted the manuscript. CR revised the manuscript. EGA performed the statistical analysis. JMB participated in the study deign and revised the manuscript. All authors read and approved the final manuscript.

\section{Acknowledgements}

We gratefully acknowledge the support of the various nurses and physicians involved in the care of the AML patients both at the PMH and the various local hospitals in and around the greater Toronto area.

\section{Author details}

1 Department of Medical Oncology and Hematology, Princess Margaret Hospital, University of Toronto, 610 University Avenue, Rm. 5-109, Toronto, ON M5G 2M9, Canada. '2Division of Infectious Diseases, University Health Network, Toronto, ON, Canada. ${ }^{3}$ Department of Biostatistics, Princess Margaret Hospital, University of Toronto, 610 University Avenue, Rm. 5-109, Toronto, ON M5G 2M9, Canada.

Received: 24 August 2012 Accepted: 19 June 2013

Published: 22 June 2013

\section{References}

1. Tallman MS: New strategies for the treatment of acute myeloid leukemia including antibodies and other novel agents. Hematology Am Soc Hematol Educ Program 2005:143-150.

2. Girmenia C, Alimena G, Latagliata R, Morano SG, Celesti F, Coppola L, Spadea A, Tosti S, Mecarocci S, D'Elia GM, et al: Out-patient management of acute myeloid leukemia after consolidation chemotherapy. Role of a hematologic emergency unit. Haematologica 1999, 84(9):814-819.

3. Gillis S, Dann EJ, Rund D: Selective discharge of patients with acute myeloid leukemia during chemotherapy-induced neutropenia. Am J Hematol 1996, 51(1):26-31.

4. Eisele L, Gunther F, Ebeling P, Nabring J, Duhrsen U, Durig J: Outpatient management of acute myeloid leukemia after intensive consolidation chemotherapy is feasible and reduces hospital treatment costs. Onkologie 2010, 33(12):658-664.

5. Savoie ML, Nevil TJ, Song KW, Forrest DL, Hogge DE, Nantel SH, Shepherd JD, Smith CA, Sutherland HJ, Toze CL, et al: Shifting to outpatient management of acute myeloid leukemia: a prospective experience. Ann Oncol 2006, 17(5):763-768.

6. Moller T, Nielsen OJ, Welinder P, Dunweber A, Hjerming M, Moser C, Kjeldsen $\mathrm{L}$ : Safe and feasible outpatient treatment following induction and consolidation chemotherapy for patients with acute leukaemia. Eur $J$ Haematol 2010, 84(4):316-322.

7. Halim TY, Song KW, Barnett MJ, Forrest DL, Hogge DE, Nantel SH, Nevill TJ, Shepherd JD, Smith CA, Sutherland HJ, et al: Positive impact of selective outpatient management of high-risk acute myelogenous leukemia on the incidence of septicemia. Ann Oncol 2007, 18(7):1246-1252.

8. Allan DS, Buckstein R, Imrie KR: Outpatient supportive care following chemotherapy for acute myeloblastic leukemia. Leuk Lymphoma 2001, 42(3):339-346.

9. Ruiz-Arguelles GJ, Apreza-Molina MG, Aleman-Hoey DD, Gomez-Almaguer D, Marin-Lopez A, Mercado-Diaz L: Outpatient supportive therapy after induction to remission therapy in adult acute myelogenous leukaemia (AML) is feasible: a multicentre study. Eur J Haematol 1995, 54(1):18-20.

10. Freifeld AG, Bow EJ, Sepkowitz KA, Boeckh MJ, Ito Jl, Mullen CA, Raad II, Rolston KV, Young JA, Wingard JR, et al: Clinical practice guideline for the use of antimicrobial agents in neutropenic patients with cancer: 2010 Update by the Infectious Diseases Society of America. Clin Infect Dis 2011, 52(4):427-431.

11. Saini L, Minden MD, Schuh AC, Yee KW, Schimmer AD, Gupta V, Atenafu EG, Murray C, Nixon S, Brandwein JM: Feasibility of outpatient consolidation chemotherapy in older versus younger patients with acute myeloid leukemia. Am J Hematol 2012, 87(3):323-326.

12. Kern W, Klose $K$, Jellen-Ritter AS, Oethinger M, Bohnert J, Kern P, Reuter $S$, von Baum H, Marre R: Fluoroquinolone resistance of Escherichia coli at a cancer center: epidemiologic evolution and effects of discontinuing prophylactic fluoroquinolone use in neutropenic patients with leukemia. Eur J Clin Microbiol Infect Dis 2005, 24(2):111-118. 
13. van Belkum A, Goessens W, van der Schee C, Lemmens-den Toom N, Vos MC, Cornelissen J, Lugtenburg E, de Marie S, Verbrugh H, Lowenberg B, et al: Rapid emergence of ciprofloxacin-resistant enterobacteriaceae containing multiple gentamicin resistance-associated integrons in a Dutch hospital. Emerg Infect Dis 2001, 7(5):862-871.

14. Bucaneve G, Micozzi A, Menichetti F, Martino P, Dionisi MS, Martinelli G, Allione B, D'Antonio D, Buelli M, Nosari AM, et al: Levofloxacin to prevent bacterial infection in patients with cancer and neutropenia. N Engl J Med 2005, 353(10):977-987.

15. Cullen M, Steven N, Billingham L, Gaunt C, Hastings M, Simmonds P, Stuart $N$, Rea D, Bower M, Fernando I, et al: Antibacterial prophylaxis after chemotherapy for solid tumors and lymphomas. N Engl J Med 2005, 353(10):988-998.

16. Leibovici L, Paul M, Cullen M, Bucaneve G, Gafter-Gvili A, Fraser A, Kern W Antibiotic prophylaxis in neutropenic patients: new evidence, practical decisions. Cancer 2006, 107(8):1743-1751.

17. Gafter-Gvili A, Fraser A, Paul M, Leibovici L: Meta-analysis: antibiotic prophylaxis reduces mortality in neutropenic patients. Ann Intern Med 2005, 142(12 Pt 1):979-995.

18. Cometta A, Calandra T, Bille J, Glauser MP: Escherichia coli resistant to fluoroquinolones in patients with cancer and neutropenia. N Engl J Med 1994, 330(17):1240-1241

19. Cattaneo C, Quaresmini G, Casari S, Capucci MA, Micheletti M, Borlenghi E, Signorini L, Re A, Carosi G, Rossi G: Recent changes in bacterial epidemiology and the emergence of fluoroquinolone-resistant Escherichia coli among patients with haematological malignancies: results of a prospective study on 823 patients at a single institution. J Antimicrob Chemother 2008, 61(3):721-728.

20. Kern W, Steib-Bauert M, de With K, Reuter S, Bertz H, Frank U, von Baum H: Fluoroquinolone consumption and resistance in haematology-oncology patients: ecological analysis in two university hospitals 1999-2002. J Antimicrob Chemother 2005, 55(1):57-60.

21. Brown NM, White LO, Blundell EL, Chown SR, Slade RR, MacGowan AP Reeves DS: Absorption of oral ofloxacin after cytotoxic chemotherapy for haematological malignancy. J Antimicrob Chemother 1993, 32(1):117-122.

22. Johnson EJ, MacGowan AP, Potter MN, Stockley RJ, White LO, Slade RR, Reeves DS: Reduced absorption of oral ciprofloxacin after chemotherapy for haematological malignancy. J Antimicrob Chemother 1990, 25(5):837-842

23. Epstein BJ, Gums JG, Drlica K: The changing face of antibiotic prescribing: the mutant selection window. Ann Pharmacother 2004, 38(10):1675-1682.

24. Rybak MJ: Pharmacodynamics: relation to antimicrobial resistance. Am J Med 2006, 119(6 Suppl 1):S37-44. discussion S62-70.

25. Reuter S, Kern WV, Sigge A, Dohner H, Marre R, Kern P, von Baum H: Impact of fluoroquinolone prophylaxis on reduced infection-related mortality among patients with neutropenia and hematologic malignancies. Clin Infect Dis 2005, 40(8):1087-1093.

26. Saito T, Yoshioka S, linuma Y, Takakura S, Fujihara N, Ichinohe T, Ishikawa T, Uchiyama T, Ichiyama S: Effects on spectrum and susceptibility patterns of isolates causing bloodstream infection by restriction of fluoroquinolone prophylaxis in a hematology-oncology unit. Eur J Clin Microbiol Infect Dis 2008, 27(3):209-216.

27. Chong Y, Yakushiji H, Ito $Y$, Kamimura T: Clinical impact of fluoroquinolone prophylaxis in neutropenic patients with hematological malignancies. International journal of infectious diseases: IJID: official publication of the International Society for Infectious Diseases 2011, 15(4):e277-281.

28. Cannas G, Pautas C, Raffoux E, Quesnel B, Botton SD, Revel TD, Reman O, Gardin C, Elhamri M, Boissel N, et al: Infectious complications in adult acute myeloid leukemia: analysis of the Acute Leukemia French Association-9802 prospective multicenter clinical trial. Leuk Lymphoma 2012, 53(6):1068-1076

29. Madani TA: Clinical infections and bloodstream isolates associated with fever in patients undergoing chemotherapy for acute myeloid leukemia. Infection 2000, 28(6):367-373.

30. Bakhshi S, Singh P, Swaroop C: Outpatient consolidation chemotherapy in pediatric acute myeloid leukemia: a retrospective analysis. Hematology 2009, 14(5):255-260.

31. Engelhard D, Elishoov H, Or R, Naparstek E, Nagler A, Strauss N, Cividalli G, Aker M, Ramu N, Simhon A, et al: Cytosine arabinoside as a major risk factor for Streptococcus viridans septicemia following bone marrow transplantation: a 5-year prospective study. Bone Marrow Transplant 1995, 16(4):565-570.
32. Okamoto Y, Ribeiro RC, Srivastava DK, Shenep JL, Pui CH, Razzouk Bl: Viridans streptococcal sepsis: clinical features and complications in childhood acute myeloid leukemia. J Pediatr Hematol Oncol 2003, 25(9):696-703.

33. Cruciani M, Malena M, Bosco O, Nardi S, Serpelloni G, Mengoli C: Reappraisal with meta-analysis of the addition of Gram-positive prophylaxis to fluoroquinolone in neutropenic patients. J Clin Oncol 2003, 21(22):4127-4137.

doi:10.1186/1471-2334-13-284

Cite this article as: Saini et al:: Ambulatory consolidation chemotherapy for acute myeloid leukemia with antibacterial prophylaxis is associated with frequent bacteremia and the emergence of fluoroquinolone resistant E. Coli. BMC Infectious Diseases 2013 13:284

\section{Submit your next manuscript to BioMed Central and take full advantage of:}

- Convenient online submission

- Thorough peer review

- No space constraints or color figure charges

- Immediate publication on acceptance

- Inclusion in PubMed, CAS, Scopus and Google Scholar

- Research which is freely available for redistribution

Submit your manuscript at www.biomedcentral.com/submit
C) Biomed Central 